\title{
Reversión de la anticoagulación en pacientes sometidos a cirugía cardiaca con circulación extracorpórea: protamina en dosis estándar vs. dosis ponderal
}

\section{Reversal of anticoagulation whith extracorporeal circulation: Standard protamine dose vs. by weight dose}

\author{
Gabriela Ramírez-González¹, Martha A. Montes-Alvarado , Álvaro J. Montiel-Jarquín ${ }^{2}$ y Arturo García-Galicia2*
}

${ }^{1}$ Servicio de Anestesiología; ${ }^{2}$ Dirección de Educación e Investigación en Salud. Unidad Médica de Alta Especialidad, Hospital de Especialidades de Puebla, Centro Médico Nacional "General de División Manuel Ávila Camacho", Instituto Mexicano del Seguro Social, Puebla, México

\begin{abstract}
Resumen
Introducción: De la dosis total de protamina aplicada para la reversión de la anticoagulación, aquella que no se une al complejo heparina/antitrombina III queda libre en el plasma. Esta tiene efectos anticoagulantes. Material y métodos: Estudio comparativo, transversal, realizado en pacientes sometidos a cirugía cardiaca con uso de circulación extracorpórea. Se administró de manera aleatorizada la dosis de protamina estándar (1.3 mg por cada 100 unidades de heparina) en el grupo A y en el grupo B se utilizó la dosis ponderal (2.4 mg protamina/kg de peso). Se valoró la efectividad de la dosis con el uso del estudio de tiempo de coagulación activado. Resultados: La media del tiempo de coagulación activado posdosis de protamina fue 129.5 y 115.2 segundos en el grupo A y B respectivamente. Sin diferencia significativa entre ambos grupos $(p>0.05)$. No hubo efectos secundarios ni complicaciones en ningún paciente. La dosis utilizada en ambos grupos ofrece reversión de la anticoagulación de manera eficaz y segura. Conclusiones: Ambas dosis de protamina ofrecen reversión eficaz y segura en pacientes sometidos a cirugía cardiaca con circulación extracorpórea.
\end{abstract}

Palabras clave: Protamina. Circulación extracorpórea. Cirugía cardiaca. Reversión de anticoagulación.

\begin{abstract}
Background: Of the total dose of protamine applied to reverse anticoagulation, that which does not bind to the heparin I antithombrin III complex (ATIII) remains free in plasma. This has anticoagulant effects. Material and methods: Comparative, cross-sectional study carried out in patients undergoing cardiac surgery with the use of extracorporeal circulation. The standard protamine dose (1.3 mg per 100 heparin units) was randomly administered in group $A$ and the weight dose (2.4 mg protamine / kg of body weight) was used in group B. Dose effectiveness was assessed with the use of the activated clotting time study. Results: The mean post-dose activated clotting time of protamine was 129.5 and 115.2 seconds in group A and $B$ respectively. No statistically significant differences between both groups ( $p>0.05)$. There were no side effects or complications in any patient.Doses used in both groups offers effective and safe anticoagulation reversal. Conclusions: Both doses of protamine offer effective and safe reversal in patients undergoing cardiac surgery with extracorporeal circulation.
\end{abstract}

Key words: Protamine. Extracorporeal circulation. Cardiac surgery. Anticoagulation reversion.

Correspondencia:

*Arturo García-Galicia

E-mail: arturo.garciaga @imss.gob.mx
Fecha de recepción: 23-09-2020

Fecha de aceptación: 07-10-2020

DOI: 10.24875/RHJM.20000114
Disponible en internet: 05-05-2021 Rev Hosp Jua Mex. 2021;88(3):111-116

www.revistahospitaljuarez.com licencia CC BY-NC-ND (http://creativecommons.org/licenses/by-nc-nd/4.0/). 


\section{Introducción}

La circulación extracorpórea (CEC) es la salida parcial del volumen sanguíneo hacia el exterior del paciente a través de una cánula venosa rígida que funciona por gravedad, así como el retorno del volumen sanguíneo al paciente impulsado por una bomba a través de una cánula arterial ${ }^{1}$

La reversión de la anticoagulación es un pilar para el éxito de la cirugía cardiaca. La reversión del efecto de la heparina se realiza con protamina en dosis de 1 a 1.3 miligramos $(\mathrm{mg})$ por cada 100 unidades $(\mathrm{U})$ de heparina; la administración rápida de la infusión se asocia con hipotensión, incluso puede ser necesaria la administración de adyuvantes hemodinámicos ${ }^{1-4}$

Sin embargo, está descrito que la dosis de protamina administrada que no se une al complejo heparina/antitrombina III (ATIII) queda libre en el plasma y tiene efectos anticoagulantes: inhibición de la trombina, trombocitopenia por activación de la cascada del complemento y disminución de la agregación plaquetaria ${ }^{5}$. También efectos adversos: anafilactoides, hipotensión arterial sistémica e hipertensión arterial pulmonar; todos estos pueden atenuarse si se administra la dosis de protamina en forma lenta y con dosis reducidas ${ }^{6}$.

Existen publicaciones con el uso de dosis a $0.8 \mathrm{mg}$ de protamina por cada $100 \mathrm{U}$ de heparina total usada, $2.4 \mathrm{mg}$ de protamina/kilogramo $(\mathrm{kg})$ de peso del paciente y $3-3.5 \mathrm{mg} / \mathrm{kg}$ de peso del paciente, independiente de la dosis de heparina usada durante la cirugía, en donde se busca disminuir las cifras de mortalidad y morbimortalidad de los pacientes sometidos a cirugía cardiaca con $\mathrm{CEC}^{7-9}$

El objetivo de este trabajo es comparar la dosis de protamina estándar con la dosis ponderal para la reversión de la anticoagulación en pacientes sometidos a cirugía cardiaca con CEC.

\section{Material y métodos}

Se trató de un estudio comparativo, transversal, analítico, unicéntrico y homodémico realizado en Hospital de Especialidades de Puebla del IMSS.

Se incluyeron pacientes cardiópatas de ambos sexos, entre 18 y 70 años, que firmaron consentimiento informado; clasificación de la American Society of Anesthesiologists (ASA) III o mayor que fueran sometidos a cirugía cardiaca con CEC tipo electiva. Se excluyeron aquellos con alergia a heparina y protamina, con trastornos de coagulación previa, con enfermedad oncológica, con obesidad mórbida o desnutrición, con cirugía previa con uso de CEC. Se eliminó a los pacientes que presentaron alguna complicación mecánica durante la cirugía (como ruptura ventricular), los sometidos a CEC > 240 minutos o pinzamiento aórtico (Pao) $>150$ minutos, los que necesitaron reheparinización para entrar en CEC por segunda vez urgente y los que fallecieron en la cirugía.

Todos los pacientes recibieron anestesia general equilibrada o total intravenosa (dependiendo de la elección de cada anestesiólogo en turno)

Se administró la dosis inicial de heparina de 300-400 $\mathrm{U}$ por $\mathrm{kg}$ de peso mediante catéter venoso central. Se tomó muestra para tiempo de coagulación activado (TCA) a los 10 minutos, que debía de ser mayor a $480 \mathrm{~s}$.

Se administró de manera aleatorizada la dosis estándar y la dosis ponderal de protamina a dos grupos. Se preparó la protamina a dosis elegida en infusión de solución salina al $0.9 \%$ de 100 mililitros durante 20 minutos.

En el grupo A se utilizó la dosis estándar (1.3 mg/100 U heparina) y en el grupo B se utilizó la dosis ponderal (2.4 mg protamina/kg de peso).

EI TCA posdosis protamina se obtuvo a los cinco minutos de terminada la infusión de protamina. Si el valor de TCA fue mayor a 150 segundos se administró una dosis de rescate de $50 \mathrm{U} / \mathrm{kg}$. Se registró el sangrado transquirúrgico y el sangrado activo al momento del egreso de quirófano.

Se realizó el seguimiento de los pacientes las primeras 24 horas en la unidad de cuidados intensivos (UCI).

Se utilizó el software Excel para la realización de la base de datos y STATA 14 y SPSS v. 25 para Windows para el análisis estadístico.

El proyecto fue aprobado por el comité local de investigación en salud número 2101 del IMSS con número de registro R-2019-2105-003.

\section{Resultados}

Se obtuvo una muestra de 20 pacientes, de los cuales 11 (55\%) fueron parte del grupo A y 9 (45\%) pacientes del grupo B. El peso promedio fue de $67.70 \mathrm{~kg}$, se puede observar la distribución del peso en la figura 1. Los diagnósticos más comunes entre los pacientes fueron valvulopatía aórtica y cardiopatía isquémica (implante valvular y revascularización). La analítica prequirúrgica se ve reflejada en la tabla 1. El euroSCORE promedio otorgado en la valoración preanestésica fue del $4.52 \%$ en el preoperatorio.

La media del TCA basal del grupo A fue de 123.27 \pm 22.13 y la del grupo $B$ fue de $113.333 \pm 7.31$; la media 




Figura 1. Distribución del peso de los pacientes incluidos en el estudio.

Tabla 1. Analítica prequirúrgica de los pacientes incluidos en el estudio

\begin{tabular}{|l|c|c|c|c|c|}
\hline & $\mathbf{n}$ & Mín & Máx & Media & DE \\
\hline Plaquetas & 20 & 132 & 362 & 229.10 & 48.318 \\
\hline $\mathrm{Hb}$ & 20 & 10.30 & 16.30 & 13.6300 & 1.30992 \\
\hline INR & 20 & 1.04 & 1.52 & 1.2465 & 0.13343 \\
\hline TP & 20 & 14.70 & 49.90 & 18.1700 & 7.54454 \\
\hline TTPa & 20 & 24.00 & 78.50 & 35.1650 & 12.45621 \\
\hline
\end{tabular}

$\mathrm{Hb}$ : hemoglobina; INR: índice internacional normalizado; TP: tiempo de protrombina; TTPa: tiempo de tromboplastina parcial activado; DE: desviación estándar; Mín: mínima; Máx: máxima.

del TCA posdosis de heparina del grupo A fue de $440.5455 \pm 25.29$ y $452 \pm 76.56$ del grupo $B$. Sin diferencia significativa entre ambos grupos $(p \geq 0.05)$.

El tiempo de CEC y el del Pao en ambos grupos presentó una media de 1.84 y 1.41 horas (Tabla 2).

Al momento de la administración de la dosis de protamina $6(30 \%)$ pacientes presentaron hipotensión arterial sistémica transitoria y $4(20 \%)$ rash, ningún paciente presentó un efecto adverso grave. Solamente el grupo A requirió dosis de rescate de protamina (Fig. 2). Dentro de las primeras 24 horas del postoperatorio un paciente falleció perteneciente al grupo $\mathrm{A}$ y un paciente tuvo que ser reintervenido del grupo $B$. Se les realizaron laboratorios a las 24 horas de su ingreso a la $\mathrm{UCl}$ (Tabla 3).

En la cuantificación del sangrado transquirúrgico se obtuvo una media de $731.81 \pm 494.10$ en el grupo A y de $661.111 \pm 295.5691$ en el grupo B; al momento del egreso de quirófano, $5(25 \%)$ pacientes presentaron sangrado activo, 4 (36.6\%) en el grupo A y 1 (11.11\%)
Tabla 2. Datos transquirúrgicos de los pacientes incluidos en el estudio

\begin{tabular}{|l|c|c|c|c|c|}
\hline & $\mathbf{n}$ & Mín & Máx & Media & DE \\
\hline Tiempo bomba & 20 & 0.33 & 2.70 & 1.8430 & 0.61482 \\
\hline Tiempo pinzamiento & 20 & 0.28 & 2.07 & 1.4130 & 0.41340 \\
\hline $\begin{array}{l}\text { Sangrado } \\
\text { transquirúrgico }\end{array}$ & 20 & 200 & 1600 & 700.00 & 408.141 \\
\hline
\end{tabular}

DE: desviación estándar; Mín: mínima; Máx: máxima.

en el grupo B. La media del sangrado a las 24 horas fue de $505.7273 \pm 359.28$ en el grupo A, por lo cual 6 $(54.5 \%)$ pacientes requirieron transfusión de hemoderivados a las 24 horas; $851.111 \pm 863.067$ en el grupo $B$, 7 (77.78\%) pacientes requirieron transfusión de hemoderivados a las 24 horas. Sin diferencia estadísticamente significativa entre ambos grupos $(p>0.05)$ (Tabla 4).

La media de la medición de TCA posdosis de protamina en el grupo A fue de $129.54 \pm 21.25$ y de 115.222 \pm 11.71 en el grupo B; sin diferencia significativa entre ambos grupos $(p>0.05)$ (Tabla 5).

\section{Discusión}

Existe una gran evolución en la cirugía cardiaca y, por ende, en su manejo anestésico. Es la obligación de todo anestesiólogo conocer las dosis adecuadas para la reversión de la anticoagulación del paciente que se somete a CEC, sin embargo, se han observado efectos adversos asociados al mal uso de la protamina, así como una alta incidencia de complicaciones posquirúrgicas en la UCl. La protamina es un polipéptido de bajo peso molecular que actualmente se utiliza para 


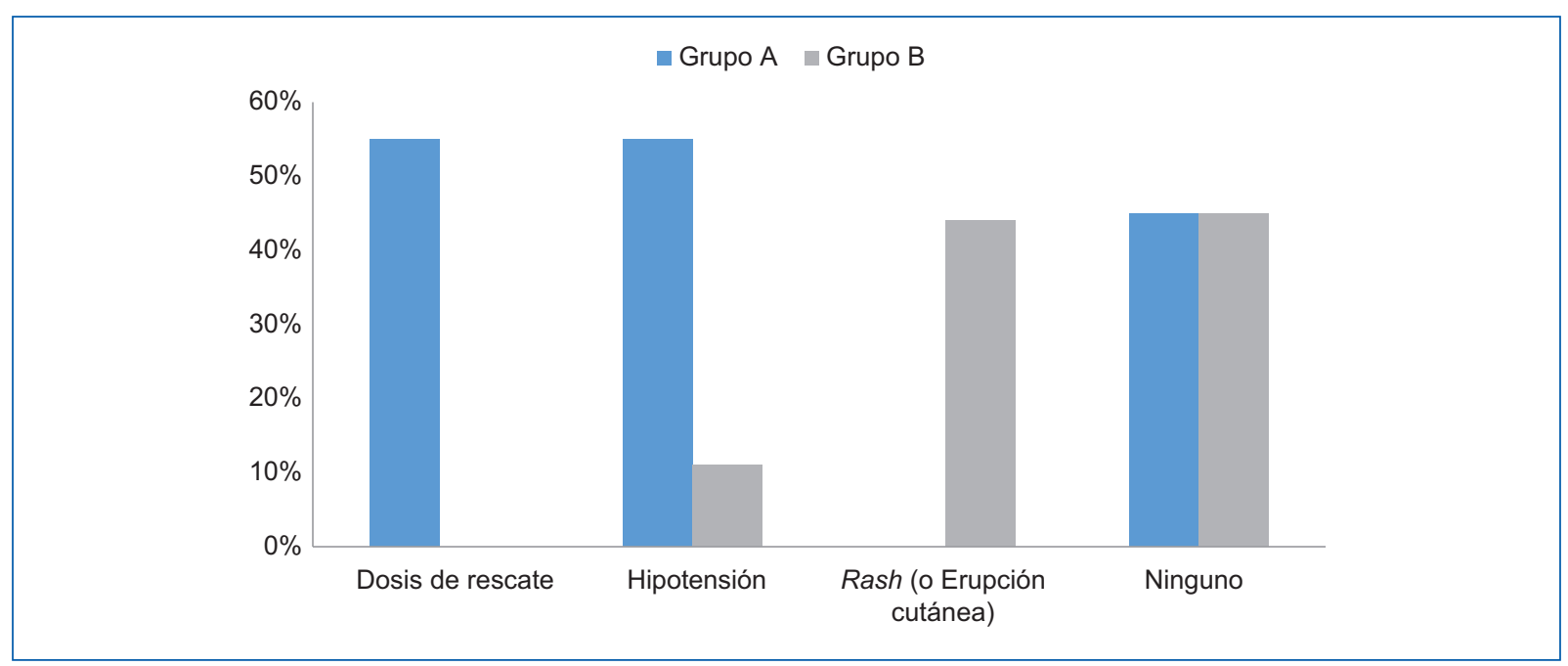

Figura 2. Distribución de necesidad de protamina de rescate y las reacciones adversas por administración en los pacientes incluidos en el estudio.

Tabla 3. Analítica posquirúrgica de los pacientes incluidos en el estudio

\begin{tabular}{|l|c|c|c|c|c|}
\hline & $\mathbf{n}$ & Mín & Máx & Media & DE \\
\hline $\mathrm{Hb}$ & 19 & 8.80 & 12.20 & 10.2842 & 1.01174 \\
\hline Plaquetas & 19 & 105 & 352 & 185 & 48.318 \\
\hline $\mathrm{Hto}$ & 19 & 28.00 & 38.00 & 31.3895 & 3.06175 \\
\hline $\mathrm{TP}$ & 19 & 10.50 & 28.60 & 19.5053 & 3.41930 \\
\hline INR & 19 & 0.70 & 1.70 & 1.4579 & 0.20757 \\
\hline TTPa & 19 & 20.40 & 42.80 & 30.2684 & 5.56008 \\
\hline
\end{tabular}

Hb: hemoglobina; Hto: hematocrito; TP: tiempo de protrombina; INR: índice internacional normalizado; TTPa: tiempo de tromboplastina parcial activado; DE: desviación estándar; Mín: mínima; Máx: máxima.

la reversión de la anticoagulación inducida por la heparina $^{10}$ En la segunda mitad del siglo $x x$ se estudiaron sus efectos ${ }^{11,12}$

El efecto revertor de la anticoagulación de la protamina es debido a que esta se une de manera irreversible a la molécula de heparina ${ }^{13}$ La neutralización de la heparina por la protamina se ve influenciada por el factor plaquetario $4^{2}$. Sin embargo, la protamina tiene efectos paradójicos ${ }^{6}$. Hay publicaciones que refieren que la dosificación incorrecta de protamina puede ocasionar «heparinización residual» y anticoagulación ${ }^{14,15}$ Es importante mencionar que el TCA se utiliza para valorar efectos anticoagulantes ${ }^{2}$. En varios protocolos
Tabla 4. Sangrado transquirúrgico (ST) a las 24 horas (S24) entre grupos A y B

\begin{tabular}{|l|c|c|c|c|c|}
\hline Variable & $\mathbf{n}$ & Media & DE & \multicolumn{2}{|c|}{ IC 95\% } \\
\hline STA & 11 & 731.8182 & 494.1016 & 399.8762 & 1063.76 \\
\hline STB & 9 & 661.1111 & 295.5691 & 433.9166 & 888.3057 \\
\hline S24A & 11 & 505.7273 & 359.2893 & 264.3534 & 747.1011 \\
\hline S24B & 9 & 851.1111 & 863.0678 & 187.6984 & 1514.524 \\
\hline $\begin{array}{l}\text { Diferencia de medias STA: grupo A - grupo B } \rightarrow \text { t: } 0.3766, \\
\text { P = 0.7108 }\end{array}$ \\
$\begin{array}{l}\text { Diferencia de medias S24: grupo A - grupo B } \rightarrow \text { t: - } 1.2108, \\
\text { P = 0 0.2416 }\end{array}$
\end{tabular}

DE: desviación estándar; IC: intervalo de confianza.

se administra como primera dosis un bolo de $300 \mathrm{U} / \mathrm{kg}$ de heparina basado en el peso del paciente ${ }^{6}$.

La dosis recomendada es de 1 a $1.3 \mathrm{mg}$ de protamina por cada 100 unidades de heparina (1:1, 1:2, 1:3 relación heparina/protamina); sin embargo, estas dosis frecuentemente son excesivas ${ }^{6} A$ estas dosis se debe esperar un TCA superior a $480 \mathrm{~s}^{16}$ Actualmente no se tiene un consenso para la titulación de la dosis de protamina ${ }^{17}$. En el trabajo realizado se utilizó la prueba de TCA para valorar la eficacia revertora de la protamina, donde el TCA basal y posdosis de heparina de ambos grupos estudiados se encontró semejante al momento de su cuantificación. 
Tabla 5. Tiempo de coagulación activado (TCA) posprotamina. Comparación entre el grupo A y el grupo B

\begin{tabular}{|l|c|c|c|c|c|}
\hline Variable & $\mathbf{n}$ & Media & DE & \multicolumn{2}{|c|}{ IC 95\% } \\
\hline TCAPPA & 11 & 129.5455 & 21.25259 & 115.2678 & 143.8231 \\
\hline TCAPPB & 9 & 115.2222 & 11.713 & 106.2188 & 124.2256 \\
\hline
\end{tabular}

DE: desviación estándar; IC: intervalo de confianza; TCAPPA: tiempo de coagulación activado posprotamina grupo A; TCAPPB: tiempo de coagulación activado posprotamina grupo $B$.

Se estudiaron 20 pacientes, al momento de su valoración preanestésica se decidió aplicar la escala de EuroSCORE, ya que su implementación como modelo predictivo para estimar el riesgo de mortalidad en cirugía cardiaca está validado en EE.UU. y Europa ${ }^{18,19}$.

La mortalidad de cirugía cardiaca con uso de CEC en México es similar a la reportada en el mundo ${ }^{20}$. El tiempo de CEC y el del Pao en ambos grupos estuvieron dentro de los rangos de seguridad establecidos por la bibliografía ${ }^{21}$.

Al momento de la administración de la dosis de protamina, $6(30 \%)$ pacientes presentaron hipotensión arterial sistémica transitoria y $4(20 \%)$ rash. La literatura refiere que después de la administración intravenosa, la protamina puede causar reacciones secundarias ${ }^{10}$. En el caso de nuestros pacientes ninguno presentó reacciones graves.

Existen publicaciones con el uso de dosis reducidas de protamina donde se busca disminuir las cifras de mortalidad y morbimortalidad de los pacientes sometidos a cirugía cardiaca con $\mathrm{CEC}^{7-9}$. En el estudio realizado por Shigeta, et al. redujo la pérdida de sangre en la cirugía cardiaca en referencia con estudios anteriores. Se confirmó la hipótesis de que la administración de dosis tituladas (mínimas) de protamina preserva la función plaquetaria en la cirugía cardiaca, ya que la sobredosis de protamina activa las plaquetas y predispone a los pacientes a un sangrado postoperatorio ${ }^{8}$. En otro estudio, realizado por Yan, et al., se realizó una comparación de dosis mínimas de protamina (tres grupos) y se analizó el sangrado postoperatorio. Se registraron los volúmenes de sangrado desde el momento de la primera dosis de protamina hasta 24 horas después de la cirugía; concluyeron que una dosis de protamina basada en dos titulaciones redujo el sangrado posquirúrgico ${ }^{9} \mathrm{El}$ sangrado postoperatorio es una de las complicaciones más comunes de la cirugía cardiaca con CEC 22 .

En el 2012 se realizó un estudio en Chile donde se compararon dos dosis distintas, una dada en forma proporcional a la heparina vs. una dosis calculada según peso del paciente. La relación protamina:heparina total fue 0.81:1 para el grupo A y 0.44:1 para el grupo $B$, donde la reversión de la heparina fue igual de eficaz en ambos grupos ${ }^{16}$. En nuestro estudio se compararon dos dosis, $1.3 \mathrm{mg}$ de protamina/ $\mathrm{U}$ heparina (1:1.3) vs. $2.4 \mathrm{mg} / \mathrm{kg}$ (1:0.44), se midió la efectividad de la reversión de la anticoagulación con el estudio de TCA y la cuantificación del sangrado.

EI TCA posdosis de protamina obtenida en ambos grupos no presentó una diferencia estadísticamente significante, ambos grupos obtuvieron la reversión de anticoagulación esperada. Es importante mencionar que dentro del grupo $\mathrm{A}$, el $45.4 \%$ requirió una dosis de rescate y en el grupo $B$ ningún paciente la requirió. Sin embargo, el $54.5 \%$ del grupo A requirió transfusión de hemoderivados para mejorar su condición, así como el $77.78 \%$ en el grupo B.

Una debilidad del estudio es que al tratarse de un estudio prospectivo que tenía un límite de tiempo de cuatro meses (donde el número de pacientes fue determinado por el periodo que duró el estudio), la pandemia por enfermedad por coronavirus 2019 (COVID-19) ocasionó que nuestro tamaño muestral para una potencia estadística mayor al $80 \%$ fuera menor.

\section{Conclusiones}

Ambas dosis son igualmente eficaces y seguras para la reversión de la anticoagulación de la heparina.

\section{Conflicto de intereses}

Los autores declaran no tener conflicto de intereses alguno.

\section{Financiamiento}

La presente investigación no ha recibido ninguna beca específica de agencias de los sectores públicos, comercial o sin ánimo de lucro.

\section{Responsabilidades éticas}

Protección de personas y animales. Los autores declaran que los procedimientos seguidos se conformaron a las normas éticas del comité de 
experimentación humana responsable y de acuerdo con la Asociación Médica Mundial y la Declaración de Helsinki.

Confidencialidad de los datos. Los autores declaran que han seguido los protocolos de su centro de trabajo sobre la publicación de datos de pacientes.

Derecho a la privacidad y consentimiento informado. Los autores han obtenido el consentimiento informado de los pacientes $y / 0$ sujetos referidos en el artículo.

\section{Bibliografía}

1. Luna Ortiz P. Anestesia cardiovascular. México: Alfil; 2013

2. Miller RD. Miller Anestesia. España: Elsevier; 2005

3. Aldrete JA. Texto de anestesiología teórico-práctica. México: Manual Moderno; 2004

4. Brück S, Skrabal C, Träger K, Reinelt $H$. Kasuistik mit Literaturübersicht - Protamingabe bei Patientin mit Fischeiweißallergie. Anasthesiol Intensivmed Notfallmedizin Schmerztherapie. 2014;49(6):360-6.

5. Despotis GJ, Joist JH, Goodnough LT. Monitoring of hemostasis in cardiac surgical patients: Impact of point-of-care testing on blood loss and transfusion outcomes. Clin Chem. 1997;43(9):1684-96.

6. Lespron-Robles MC, Molina-Méndez FJ. Terapia anticoagulante en la circulación extracorpórea. Arch Cardiol Mex. 2007;77(Suppl. 4):185-93.

7. Carrillo-Esper R, de los Monteros-Estrada IE, Rosales-Gutiérrez AO, Zepeda-Mendoza AD, Alonso-Martínez D, Sánchez-Moreno MA, et al. Concentrado de complejo protrombínico en el perioperatorio. Rev Mex Anestesiol. 2015;38(1):35-43.

8. Shigeta O, Kojima H, Hiramatsu Y, Jikuya T, Terada Y, Atsumi N, et al Low-dose protamine based on heparin-protamine titration method reduces platelet dysfunction after cardiopulmonary bypass. J Thorac Cardiovasc Surg. 1999;118(2):354-60.

9. Guo Y, Tang J, Du L, Liu J, Liu RC, Liu X, et al. Protamine dosage based on two titrations reduces blood loss after valve replacement surgery: A prospective, double-blinded, randomized study. Can J Cardiol. 2012;28(5):547-52.
10. Madani H, Sadiki EO, Bouziane M, Amaarouch S, Madani M, Khatouf M. L'allergie à la protamine en chirurgie cardiovasculaire. Ann Pharm Fr. 2014;72(3):143-5.

11. Boer C, Meesters $\mathrm{Ml}$, Veerhoek $\mathrm{D}$, Vonk ABA. Anticoagulant and side-effects of protamine in cardiac surgery: a narrative review. $\mathrm{Br} \mathrm{J}$ Anaesth. 2018;120(5):914-27.

12. Butterworth J, Lin YA, Prielipp R, Bennett J, James R. The pharmacokinetics and cardiovascular effects of a single intravenous dose of protamine in normal volunteers. Anesth Analg. 2002;94(3):514-22.

13. Shanberge JN, Quattrociocchi-Longe TM. Influence of platelet factor 4 on the neutralization of heparin by protamine. Ann N Y Acad Sci. 1989;556(1):354-65.

14. Meesters MI, Veerhoek D, de Lange F, de Vries JW, de Jong JR, Romiin JWA, et al. Effect of high or low protamine dosing on postoperative bleeding following heparin anticoagulation in cardiac surgery a randomised clinical trial. Thromb Haemost. 2016;116 (2):251-61.

15. Taneja R, Marwaha G, Sinha P, Quantz M, Stitt L, Gao R, et al. Elevated activated partial thromboplastin time does not correlate with heparin rebound following cardiac surgery. Can J Anesth. 2009;56(7):489-96.

16. Luciano GJ, Mauricio RG, Mariana VR, Roberto ME, Marcela ÁA, Renato $\mathrm{CA}$, et al. Reversión de la anticoagulación en pacientes con dosis altas de heparina: Protamina en dosis standard versus dosis reducidas. Rev Chil Anest. 2012;41(2):108-12.

17. Kunz SA, Miles LF, lanno DJ, Mirowska-Allen KL, Matalanis G, Bellomo R, et al. The effect of protamine dosing variation on bleeding and transfusion after heparinisation for cardiopulmonary bypass. Perfus (United Kingdom). 2018;33(6):445-52.

18. Rodríguez-Chávez LL, Figueroa-Solano J, Muñoz-Consuegra CE, Avila-Vanzzini N, Kuri-Alfaro J. EuroSCORE subestima el riesgo de mortalidad en cirugía cardiaca valvular de población mexicana. Arch Cardiol México. 2017;87(1):18-25.

19. Riera-M, Carrillo-A, Ibáñez-J, Sáez-de-Ibarra-JI, Fiol M, Bonnin YO. VaIor predictivo del modelo EuroSCORE en la cirugía cardíaca de nuestro centro. Med Intensiva. 2007;31(5):231-6.

20. Rodríguez-Hernández A, García-Torres M, Bucio-Reta E, Baranda-Tovar FM. Análisis de mortalidad y estancia hospitalaria en cirugía cardiaca en México 2015: datos del Instituto Nacional de Cardiología. Arch Cardiol México. 2018;88(5):397-402.

21. Morales-Pogoda II. Asociación entre el tiempo de circulación extracorpórea y pinzamiento aórtico en la mortalidad de pacientes operados de cirugía cardiaca. Rev Sanid Milit. 2014;68(5):251-6.

22. Izaguirre-ávila R, Evelyn D, Rosa C, García-Espinosa Jl, Grimaldo-gómez FA. Hemorragia crítica en cirugía cardíaca con circulación extracorpórea. Rev Mex Anestesiol. 2017;40(6):405-8. 